\title{
Biomarker alterations associated with distinct patterns of metastatic spread in colorectal cancer
}

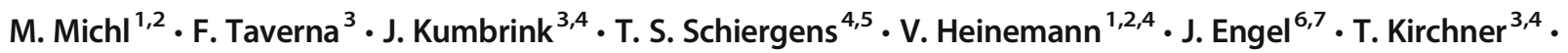 \\ Jens Neumann ${ }^{3,4}$ (B)
}

Received: 18 September 2020 / Revised: 25 November 2020 / Accepted: 1 December 2020 / Published online: 9 December 2020

(C) The Author(s) 2020

\begin{abstract}
Metastatic spread is the most important life-threatening feature of colorectal cancer and is supposed to be mainly driven by alterations in different carcinogenic pathways. The present study compared mutation and expression profiles of distinctive biomarkers in colorectal cancer patients with different clinical metastatic patterns. As for a case-control study, patients were matched according to T category, grading and primary tumour site. Overall, 246 patients with either exclusive lung metastasis $(N$ $=82)$, exclusive liver metastasis $(N=82)$ or non-metastatic colorectal cancer $(N=82)$ were identified. Paraffin-embedded specimens were examined for mutations in the $R A S$ and $R A F$ genes and for the expression of $\beta$-catenin and CD133. Clinical endpoints were presence or absence of distant metastasis, formation of metastasis in lungs versus the liver and survival. MAPK pathway mutations in either the KRAS, NRAS or $B R A F$ gene were associated with the development of lung metastasis $(63.4 \%)$ compared to the control group $(47.6 \% ; p=0.04)$. MAPK pathway alterations plus high $\beta$-catenin expression were associated with metastasis to the lungs but not to the liver $(28.0 \%$ vs. $13.4 \% ; p=0.02)$. High CD133 expression correlated with the development of liver metastasis compared to the control group $(30.5 \%$ vs. $14.6 \% ; p=0.02)$. This data indicates that different patterns of distant spread are associated with specific biomarker alterations and may represent different molecular subtypes of colorectal cancer. However, underlying mechanisms of metastasis formation in different anatomic sites remains unclear. Since knowledge of the anticipated site of distant spread would substantially impact clinical management, further research is needed to identify solid biomarkers for different metastatic patterns.
\end{abstract}

Keywords Lung metastasis $\cdot$ Metastatic colorectal cancer $\cdot$ Biomarker $\cdot$ CD133 $\cdot \beta$-catenin $\cdot$ MAPK pathway mutations

Jens Neumann

jens.neumann@med.uni-muenchen.de

1 Department of Medicine III, University Hospital, LMU Munich, München, Germany

2 Comprehensive Cancer Center, University Hospital, LMU Munich, München, Germany

3 Institute of Pathology, Faculty of Medicine, LMU Munich, Thalkirchner Str. 36, D-81377 München, Germany

4 German Cancer Consortium (DKTK), German Cancer Research Centre (DKFZ), Heidelberg, Germany

5 Department of General, Visceral and Transplantation Surgery, University Hospital, LMU Munich, München, Germany

6 Munich Cancer Registry (MCR), Ludwig-Maximilians-University of Munich, Munich, Germany

7 Institute of Medical Informatics, Biometry and Epidemiology (IBE), Ludwig-Maximilians-University of Munich, München, Germany

\section{Introduction}

Organ metastasis is still the leading cause of death in the majority of colorectal cancer (CRC) patients. Prognosis greatly differs inter-individually and crucially depends-interalia - on metastatic patterns [1]. Organs most commonly affected are the liver (50-70\%) and lungs (10-30\%) [2]. Today, management of metastatic CRC (mCRC) includes multidisciplinary approaches combining surgery, systemic therapy and local ablative techniques in order to provide personalized treatment procedures [3-6]. Thus, a reasonable effort was put into the identification of prognostic markers for predicting the clinical course and individual prognosis of patients with mCRC [7-9]. The RAS and BRAF mutational status as well as the mismatch repair (MMR) status represent acknowledged biomarkers in $\mathrm{mCRC}$ patients as they have a prognostic impact and influence systemic treatment [10-12]. Besides its prognostic and predictive relevance, the RAS mutational status 
was shown to impact the metastatic phenotype in mCRC [13]. Whilst the presence of a $R A S$ mutation seems to constitute an independent risk factor for the development of lung, brain and bone metastasis, $R A S$ wild-type tumours are associated with the presence of liver metastasis [14-18].

However, apart from these analyses, no established biomarkers exist for predicting the formation of metastasis in the lungs or the liver to date. In previous studies, our group demonstrated that the expression of CD133 and, in particular, the combined expression of CD133 and $\beta$-catenin, both associated with the Wnt-/ $\beta$-catenin-pathway and stem cell features of tumour cells, significantly correlates with poor prognosis as well as the formation of distant metastasis in the liver [19-21]. Further analyses revealed that the expression of these markers did not correlate with the presence of peritoneal carcinomatosis or brain metastasis [22, 23]. We hypothesized that different mechanisms must play a role in the development of cavitary metastasis, brain metastasis and metastases in the liver. On that basis, the question arose whether both markers could also play a crucial role in the development of pulmonary metastasis.

Within the present study, we examined CRC specimens from patients with exclusive lung metastasis (PUL group) and exclusive liver metastasis (HEP group) and compared them with tumours deriving from patients without distant metastases that served as the control group (M0 group). Based on the data from the literature and the results from our study group, we focused on the investigation of single biomarkers consisting of MAP kinase pathway mutations as well as $\beta$ catenin and CD133 expression status and analyzed their impact on the formation of lung or liver metastasis and survival. Furthermore, the association of different biomarker combinations with different localizations of distant metastases was evaluated.

As most data in the literature is deducted from retrospectiveexploratory analyses or epidemiological studies, we specifically chose a case-control format as study design in order to eliminate competing for confounding factors by matching all study patients according to the most relevant clinical and pathological criteria in CRC.

\section{Material and methods}

\section{Patients}

All patients involved in the present analysis were diagnosed at the Institute of Pathology, Faculty of Medicine, LMU Munich, and subsequently identified via systematic database search in collaboration with the Munich Cancer Registry (MCR). The MCR covers an estimated population of meanwhile 4.9 million inhabitants in the southern part of Germany. Search items comprised "colorectal cancer" and "exclusive lung metastasis" (for the creation of the PUL group), "colorectal cancer" and "exclusive liver metastasis" (for the generation of the HEP group) and "colorectal cancer" and "no organ metastasis" and "no local recurrence" within 5 years after first CRC diagnosis (for the formation of the M0 group). Patients with a histologically proven diagnosis of CRC and the histological or radiological diagnosis of lung or liver metastasis reported to the MCR between 1998 and 2017 were considered for the PUL or HEP group, respectively. As a control, the M0 group consisting of CRC patients with nonmetastatic disease reported to the MCR between 1998 and 2012 was assembled. By halting the recruitment of patients for the M0 group in 2012, non-metastatic and recurrence-free survival during a follow-up period of at least 5 years was ensured. Available patient and tumour characteristics as well as survival data were collected. Patients with secondary malignancies were excluded.

\section{Study design}

A matched-pair analysis was deemed appropriate for the present investigation. Patients from all groups were matched according to $\mathrm{pT}$ category, grading and primary tumour site. As suitable for a matched-pair analysis, all groups consisted of equal patient numbers. Availability of sufficient analyzable tumour tissue limited patient numbers to 82 patients per group (Online Resource 1).

\section{Immunohistochemistry}

For immunohistochemistry, $5-\mu \mathrm{m}$ whole-tissue sections of formalin-fixed and paraffin-embedded (FFPE) tumour samples were stained employing a Ventana Benchmark (Ventana Medical Systems, Oro Valley, AZ) following the manufacturer's instructions. A detailed description of antibodies and protocols used in this study is provided in Online Resource 2. To exclude unspecific reactions of antibodies and/or reagents, isotype and system controls were performed.

\section{Scoring of immunohistochemistry}

All samples were evaluated independently by two investigators (J.N. and F.T.), both blinded for the clinical outcome. In case of discrepancy, samples were jointly reviewed and a consensus was reached. A staining score for nuclear expression of $\beta$-catenin was based on the quantity of stained tumour cell nuclei throughout the whole tumour, whereas the intensity of staining was not considered (Fig. 1a and b). The score was as follows: 0 , negative; $1+,<30 \% ; 2+, 30-60 \% ; 3+,>$ $60 \%$ positive cells. Subsequently, the cases were classified into low (scores 0 and 1) and high-grade (scores 2 and 3) expression. For CD133, protein expression was defined as either staining of apical membranous parts of the cells or of shed cellular debris in the tumour glands. CD133 expression 
levels were scored as low grade $(<50 \%$ of positive tumour glands, Fig. 1c) or high grade ( $\geq 50 \%$ positive tumour glands, Fig. 1d). Aberrant expression of p53 was defined as strong diffuse nuclear staining in $>90 \%$ of tumour cells (Fig. 1e) or complete absence of p53 expression in all tumour cells (Fig. 1f). A regulated p53 expression pattern is shown in Fig. 1g. Loss of MLH1 and MSH2 expression (reflecting a dMMR) was recorded when nuclear staining was absent in malignant cells but preserved in the stroma cells or normal epithelial cells, respectively. Cases with preserved nuclear MLH1 and MSH2 expression in tumour cells were classified as cases with proficient MMR status (Fig. 1h-j).

\section{Mutation analysis}

For enrichment of tumour tissue, H\&E-stained histological serial sections were inspected and areas containing tumour cells were defined and marked. These sections were used as blueprints for transferring the marked areas onto unstained dewaxed tissue sections. Then, marked areas were microdissected under microscopic control using scalpel blades. From the resulting tissue, DNA was isolated using QIAamp ${ }^{\circledR}$ DNA Micro Kit (Qiagen, Hilden, Germany) following the user's handbook. For the detection of BRAF, KRAS and NRAS mutations, pyrosequencing was employed. Briefly, HotStarTaq ${ }^{\circledR}$ Polymerase (Qiagen, Hilden, Germany) was used together with $1 \times$ PCR buffer $\left(1.5 \mathrm{mM} \mathrm{MgCl}_{2}\right), 200 \mu \mathrm{M}$ dNTPs and 400-nM primers applying optimized PCR conditions (Online Resource 3). PCR products were analyzed using PyroMark ${ }^{\circledR}$ Gold Q24 kits (Qiagen, Hilden, Germany) together with $0.3 \mu \mathrm{M}$ of the corresponding sequencing primer (Online Resource 3) employing the PyroMark ${ }^{\circledR}$ Q24 device (Qiagen, Hilden, Germany). Finally, data were analyzed applying the PyroMark ${ }^{\circledR}$ Q24 software (Qiagen, Hilden, Germany).

\section{Statistical analysis}

For comparison of patient and tumour characteristics between groups, a two-sided $\chi^{2}$ test was used. Only "age" as a metric and not normally distributed variable was tested with the Kruskal-Wallis test (global $p$ ) and the Mann-Whitney $U$ test (PUL vs. HEP). For global testing and head-to-head comparisons, the significance of correlations of single biomarkers and biomarker combinations was calculated using a $\chi^{2}$ test. Survival analysis for overall and progression-free survival was assessed using the Kaplan-Meier method and univariate survival distributions were compared using the log-rank test. Multivariate logistic regression analysis was performed in order to rule out confounding factors between groups. For multivariate analysis, the factors "age", "lymph node status" (N0 vs. N+), "MAP kinase status", "MMR status", "p53 expression status", "beta-catenin expression status" and "CD133 expression status" were considered. For all statistical tests, the SPSS V. 26.0 Software (IBM Inc., Armonk, NY) was employed. A $p$ value lower than 5\% $(p<0.05)$ was considered statistically significant.

\section{Results}

\section{Patient and tumour characteristics}

The entire study population consisted of 246 patients (145 male (59\%), 101 female (41\%)) with histologically proven adenocarcinoma of the colorectum and exclusive lung metastasis (PUL; $N=82$ ), exclusive liver metastasis (HEP; $N=82$ ) or non-metastatic disease (M0; $N=82)$. Baseline patient demographics and tumour characteristics of the analyzed patient cohort are presented in Table 1 and Online Resource 4. Patients differed between groups in a few non-matching variables such as gender, primary tumour site and $\mathrm{pN}$ category (global p). However, when comparing only patients with exclusive lung versus exclusive liver metastasis (PUL vs. HEP), baseline characteristics were equally distributed.

Median age at first CRC diagnosis was significantly higher in patients of the M0 group compared to the PUL and HEP groups ( 71.3 vs. 64.4 vs. 63.5 years; global $p<0.001)$. A higher proportion of patients from the M0 group presented with a primary tumour located in the colon $(51(62.2 \%))$, whereas in the PUL and the HEP group, the majority of primaries was found in the rectum (50 (61.0\%) and $40(48.8 \%))$ (global $p<0.001$ ). Detailed subgroup analysis showed that more patients from the PUL group had their primary in the rectum compared to the M0 group $(p<0.001)$ and also more cases with rectal primaries were found in the HEP group compared to the M0 group $(p=0.01)$. No significant difference with regard to the primary tumour site was detected between the PUL and the HEP group $(p=0.29)$. Also, the temporal occurrence of metastasis was significantly different between groups. Whilst the majority of liver metastasis occurred synchronously (59 (72.0\%)), lung metastasis was detected synchronously and metachronously in nearly equal parts (42 $(51.2 \%)$ and 40 (48.8\%); $p=0.002$ for PUL vs. HEP).

\section{Frequency of single-biomarker alterations according to metastatic status}

For each patient group, all detected MAP kinase (MAPK) pathway mutations and immunohistochemical expression profiles of CD133, $\beta$-catenin and $\mathrm{p} 53$ are listed in Table 2 . Analyzed MAPK pathway alterations included the $R A S \mathrm{mu}-$ tational status of the KRAS and NRAS hotspots in exons 2, 3 and 4 as well as the $B R A F$ V600 mutational status and other $B R A F$ mutations. In decreasing order of frequency, MAPK pathway alterations were detected in the PUL (52 (63.4\%)), 

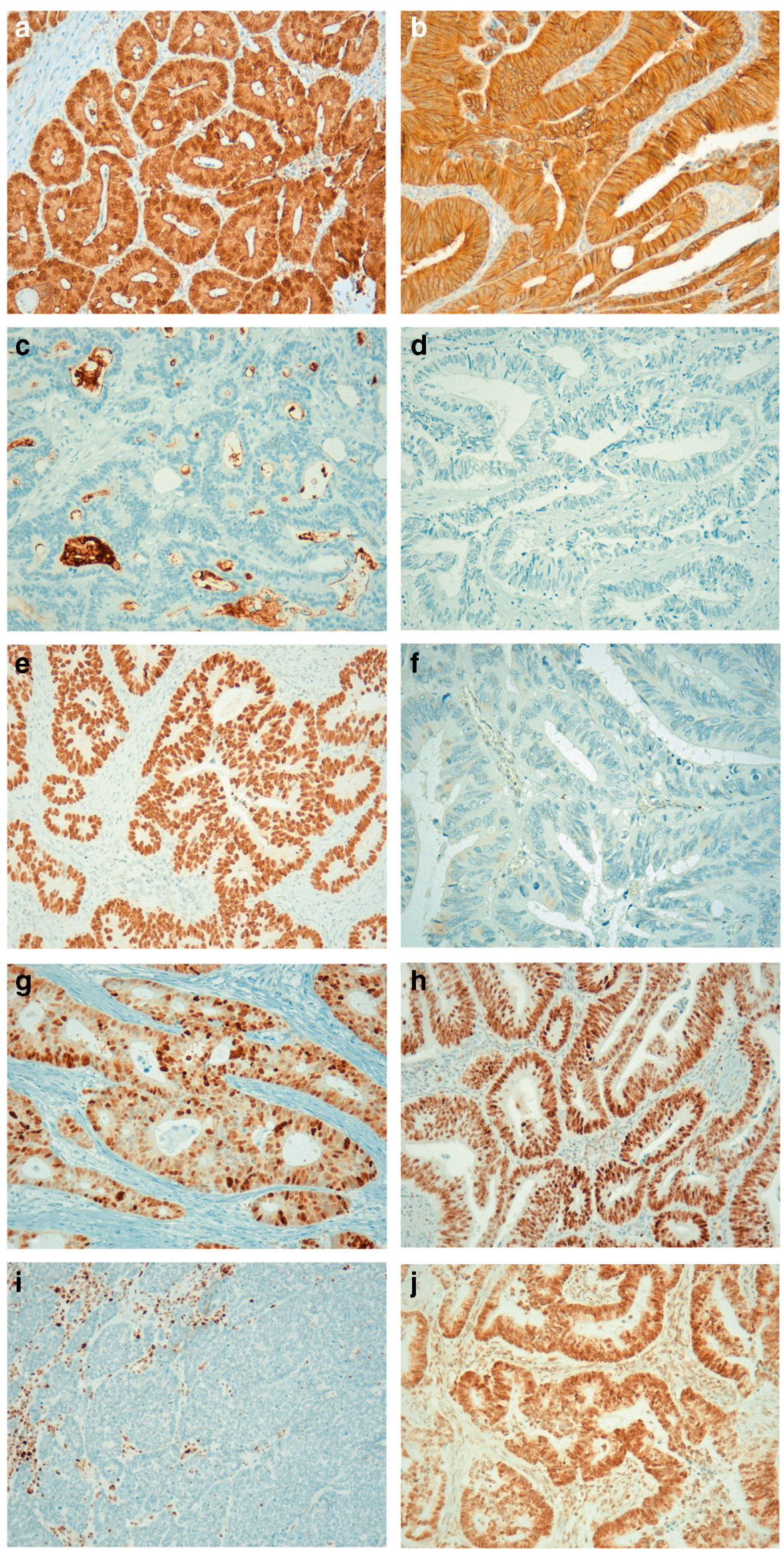
Fig. 1 Immunohistochemical analysis in colorectal cancer specimens. a High nuclear $\beta$-catenin expression; b low nuclear $\beta$-catenin expression; c high CD133 expression; d low CD133 expression; e deregulated p53 expression pattern with diffuse strong nuclear $\mathrm{p} 53$ staining; $\mathbf{f}$ deregulated p53 expression pattern with the absence of nuclear p53 expression; $\mathbf{g}$ regulated p53 expression pattern; $\mathbf{h}$ positive MLH1-staining (pMMR); $\mathbf{i}$ negative MLH1-staining (dMMR); j positive MSH2-staining (pMMR)

HEP $(42(51.2 \%))$ and M0 (39 $(47.6 \%))$ group (global $p=$ $0.10)$. Thus, the highest frequency of MAPK pathway mutations was observed in patients with lung metastasis mainly consisting of $R A S$ mutations $(N=48(58.5 \%))$ and hereunder especially of $K R A S$ exon 2 mutations $(N=42(51.2 \%))$. Consistently, in the HEP and M0 group, $R A S$ respectively $K R A S$ exon 2 mutations represented the most common MAPK pathway mutations (HEP (41 RAS (50.0\%) and 38 KRAS exon 2 mutations (46.3\%)); M0 (35 RAS (42.7\%) and 33 KRAS exon 2 mutations (40.2\%)). One patient with liver metastasis was diagnosed with a double mutation in both the $K R A S$ exon 3 and the NRAS exon 2, respectively. No difference in frequencies of $N R A S$ and $B R A F$ mutations was observed between groups ( $p=0.60$ and $p=0.25$, respectively).
Table 1 Patient and tumour characteristics of the entire study population $(N=246)$

\begin{tabular}{|c|c|c|c|c|c|}
\hline \multirow[t]{2}{*}{ Total $N=246$} & \multirow[t]{2}{*}{ PUL $N=82$} & \multirow[t]{2}{*}{$\operatorname{HEP} N=82$} & \multirow[t]{2}{*}{ M0 $N=82$} & \multicolumn{2}{|l|}{$P$} \\
\hline & & & & Global & PUL vs HEP \\
\hline \multicolumn{6}{|l|}{ Sex } \\
\hline Female & $27(32.9)$ & $32(39.0)$ & $42(51.2)$ & \multirow[t]{2}{*}{0.053} & \multirow[t]{2}{*}{0.42} \\
\hline Male & $55(67.1)$ & $50(61.0)$ & $40(48.8)$ & & \\
\hline \multicolumn{6}{|c|}{ Age at first diagnosis of CRC } \\
\hline Median, years & 64.4 & 63.5 & 71.3 & $<0.001$ & 0.28 \\
\hline$\geq 70$ years & $28(34.1)$ & $24(29.3)$ & $46(56.1)$ & \multirow{3}{*}{$\begin{array}{l}0.001 \\
<0.001\end{array}$} & \multirow{3}{*}{$\begin{array}{l}0.50 \\
0.75\end{array}$} \\
\hline$\geq 65$ years & $39(47.6)$ & $37(45.1)$ & $64(78.0)$ & & \\
\hline$<65$ years & $43(52.4)$ & $45(54.9)$ & $18(22.0)$ & & \\
\hline \multicolumn{6}{|l|}{ Sidedness of primary } \\
\hline Right colon & $9(11.0)$ & $9(11.0)$ & $9(11.0)$ & \multirow[t]{2}{*}{1.00} & \multirow[t]{2}{*}{1.00} \\
\hline Left colon & $73(89.0)$ & $73(89.0)$ & $73(89.0)$ & & \\
\hline \multicolumn{6}{|l|}{ Primary tumour site } \\
\hline Colon & $25(30.5)$ & $32(39.0)$ & $51(62.2)$ & \multirow[t]{3}{*}{$<0.001$} & \multirow[t]{3}{*}{0.29} \\
\hline Rectosigmoid & $7(8.5)$ & $10(12.2)$ & $7(8.5)$ & & \\
\hline Rectum & $50(61.0)$ & $40(48.8)$ & $24(29.3)$ & & \\
\hline \multicolumn{6}{|l|}{ Grading } \\
\hline Low grade $(\mathrm{G} 1, \mathrm{G} 2)$ & $54(65.9)$ & $56(68.3)$ & $56(68.3)$ & \multirow[t]{3}{*}{0.97} & \multirow[t]{3}{*}{0.59} \\
\hline High grade (G3) & $27(32.9)$ & $26(31.7)$ & $26(31.7)$ & & \\
\hline Unknown & $1(1.2)$ & - & - & & \\
\hline \multicolumn{6}{|l|}{ pT stage } \\
\hline pT0 & $2(2.4)$ & - & - & & \\
\hline pT1 & $3(3.7)$ & $4(4.9)$ & $4(4.9)$ & & \\
\hline pT2 & $11(13.4)$ & $11(13.4)$ & $8(9.8)$ & \multirow[t]{4}{*}{0.50} & \multirow[t]{4}{*}{0.49} \\
\hline pT3 & $57(69.5)$ & $58(70.7)$ & $60(73.2)$ & & \\
\hline pT4 & $7(8.5)$ & $9(11.0)$ & $10(12.2)$ & & \\
\hline Unknown & $2(2.4)$ & - & - & & \\
\hline \multicolumn{6}{|l|}{ pN status } \\
\hline pN0 & $32(39.0)$ & $31(37.8)$ & $52(63.4)$ & & \\
\hline $\mathrm{pN} 1$ & $31(37.8)$ & $37(45.1)$ & $26(31.7)$ & \multirow[t]{3}{*}{0.001} & \multirow[t]{3}{*}{0.14} \\
\hline $\mathrm{pN} 2$ & $14(17.1)$ & $14(17.1)$ & $3(3.7)$ & & \\
\hline Unknown & $5(6.1)$ & - & $1(1.2)$ & & \\
\hline \multicolumn{6}{|l|}{ Time of metastasis } \\
\hline Metachronous & $42(51.2)$ & $23(28.0)$ & NA & \multirow[t]{2}{*}{-} & 0.002 \\
\hline Synchronous & $40(48.8)$ & $59(72.0)$ & NA & & \\
\hline
\end{tabular}

For each category, absolute patient numbers are given and percentages in brackets. NA not applicable 
Table 2 Frequency of MAP kinase mutations and immunohistochemical expressions of CD133, beta-catenin and p53 comparing the three patient cohorts

\begin{tabular}{|c|c|c|c|c|c|c|c|c|}
\hline & Total $N=246$ & \multicolumn{2}{|c|}{ PUL $N=82$} & \multicolumn{2}{|c|}{$\operatorname{HEP} N=82$} & \multicolumn{2}{|l|}{ M0 $N=82$} & Global $P$ \\
\hline \multicolumn{9}{|c|}{ MAP kinase mutational status } \\
\hline $\begin{array}{l}\text { MAP kinase mutation } \\
\text { MAP kinase wild type }\end{array}$ & $\begin{array}{l}133 \\
113\end{array}$ & \multicolumn{2}{|l|}{$\begin{array}{l}52(63.4) \\
30(36.6)\end{array}$} & \multicolumn{2}{|l|}{$42(51.2)$} & \multicolumn{2}{|l|}{$39(47.6)$} & 0.10 \\
\hline $\begin{array}{l}\text { RAS mutation } \\
\text { RAS wild type }\end{array}$ & $\begin{array}{l}124 \\
122\end{array}$ & \multicolumn{2}{|l|}{$\begin{array}{l}48(58.5) \\
34(41.5)\end{array}$} & \multicolumn{2}{|l|}{$\begin{array}{l}41(50.0) \\
41(50.0)\end{array}$} & \multicolumn{2}{|l|}{$\begin{array}{l}35(42.7) \\
47(57.3)\end{array}$} & 0.13 \\
\hline $\begin{array}{l}\text { KRAS mutation } \\
\text { Exon } 2\end{array}$ & $\begin{array}{l}117 \\
103\end{array}$ & \multicolumn{2}{|l|}{$\begin{array}{l}46(56.1) \\
42(51.2)\end{array}$} & \multicolumn{2}{|l|}{$38(46.3)$} & $33(40.2)$ & & 0.12 \\
\hline Exon 3 & 8 & \multicolumn{2}{|l|}{$1(1.2)$} & \multicolumn{2}{|l|}{$4(4.9)^{\#}$} & \multicolumn{3}{|l|}{$3(3.7)$} \\
\hline Exon 4 & 6 & \multicolumn{2}{|l|}{$3(3.7)$} & \multicolumn{2}{|l|}{$1(1.2)$} & \multicolumn{3}{|l|}{$2(2.4)$} \\
\hline KRAS wild type & 129 & \multicolumn{2}{|l|}{$36(43.9)$} & \multicolumn{2}{|l|}{$44(53.7)$} & \multicolumn{2}{|l|}{$49(59.8)$} & \\
\hline $\begin{array}{l}\text { NRAS mutation } \\
\text { Exon } 2\end{array}$ & $\begin{array}{l}8 \\
5\end{array}$ & \multicolumn{2}{|l|}{$2(2.4)$} & \multicolumn{2}{|l|}{$4(4.9)$} & \multicolumn{2}{|l|}{$2(2.4)$} & 0.60 \\
\hline Exon 3 & 3 & $1(1.2)$ & & $2(2.4)$ & & - & & \\
\hline Exon 4 & - & - & & - & & - & & \\
\hline NRAS wild type & 238 & $80(97.6)$ & & $78(95.1)$ & & $80(97.6)$ & & \\
\hline $\begin{array}{l}\text { BRAF mutation } \\
\text { V600E }\end{array}$ & $\begin{array}{l}9 \\
8\end{array}$ & $\begin{array}{l}4(4.9) \\
4(4.9)\end{array}$ & & $\begin{array}{l}1(1.2) \\
1(1.2)\end{array}$ & & $\begin{array}{l}4(4.9) \\
3(3.7)\end{array}$ & & 0.35 \\
\hline Other & 1 & - & & - & & $1(1.2)$ & & \\
\hline BRAF wild type & 237 & $78(95.1)$ & & $81(98.8)$ & & $78(95.1)$ & & \\
\hline Stem cell marker & & & & & & & & \\
\hline $\begin{array}{l}\text { CD133 high } \\
\text { CD133 low }\end{array}$ & $\begin{array}{l}57 \\
189\end{array}$ & $\begin{array}{l}20(24.4) \\
62(75.6)\end{array}$ & & $\begin{array}{l}25(30.5) \\
57(69.5)\end{array}$ & & $\begin{array}{l}12(14.6) \\
70(85.4)\end{array}$ & & 0.05 \\
\hline Wnt pathway & & & & & & & & \\
\hline $\begin{array}{l}\beta \text {-catenin high } \\
\beta \text {-catenin low }\end{array}$ & $\begin{array}{l}109 \\
137\end{array}$ & $\begin{array}{l}41(50.0) \\
41(50.0)\end{array}$ & & $\begin{array}{l}33(40.2) \\
49(59.8)\end{array}$ & & $\begin{array}{l}35(42.7) \\
47(57.3)\end{array}$ & & 0.43 \\
\hline p53 pathway & & & & & & & & \\
\hline $\begin{array}{l}\text { p53 regulated } \\
\text { p53 deregulated }\end{array}$ & $\begin{array}{l}80 \\
166\end{array}$ & $\begin{array}{l}28(34.2) \\
54(65.8)\end{array}$ & & $\begin{array}{l}24(29.3) \\
58(70.7)\end{array}$ & & $\begin{array}{l}28(34.2) \\
54(65.8)\end{array}$ & & 0.74 \\
\hline MMR status & & & & & & & & \\
\hline $\begin{array}{l}\text { pMMR } \\
\text { dMMR }\end{array}$ & $\begin{array}{l}239 \\
7\end{array}$ & $\begin{array}{l}81(98.8) \\
1(1.2)\end{array}$ & & $\begin{array}{l}80(97.6) \\
2(2.4)\end{array}$ & & $\begin{array}{l}78(95.1) \\
4(4.9)\end{array}$ & & 0.36 \\
\hline Marker combinations* & & Positive & Negative & Positive & Negative & Positive & Negative & \\
\hline MAP kinase mutational & JS $\beta$-catenin expression & $23(28.0)$ & $59(72.0)$ & $11(13.4)$ & $71(86.6)$ & $14(17.1)$ & $68(82.9)$ & 0.048 \\
\hline MAP kinase mutational & JS CD133 expression & $15(18.3)$ & $67(81.7)$ & $17(20.7)$ & $65(79.3)$ & $5(6.1)$ & 77 (93.9) & 0.02 \\
\hline$\beta$-catenin expression PL & 3 expression & $9(11.0)$ & $73(89.0)$ & $10(12.2)$ & $72(87.8)$ & $8(9.8)$ & $74(90.2)$ & 0.88 \\
\hline
\end{tabular}

For each category, absolute patient numbers are given and percentages in brackets. Significant $p$ values are printed in italics

*Positive is defined as both markers being positive (positive for MAP kinase mutational status is defined as the presence of a mutation; positive for $\beta$ catenin and CD133 expression is defined as a high expression). Negative is defined as one of the markers or both markers being negative (negative for MAP kinase mutational status is defined as wild type; negative for $\beta$-catenin and CD133 expression is defined as a low expression)

${ }^{\#}$ One case with a double mutation in KRAS Exon 3 and NRAS Exon 2

Regarding the stem cell marker CD133, high immunohistochemical expression was most frequently detected in patients with liver metastasis $(N=25(30.5 \%))$, nearly reaching the level of significance in comparison with the PUL and M0 group $(p=0.05)$. Immunohistochemical detection of nuclear $\beta$-catenin expression representing an active $\mathrm{Wnt} / \beta$-catenin pathway did not reveal significant differences between groups as a single biomarker. The p53 status did not differ significantly between groups and was even considered almost identical. Seven out of 246 patients (2.9\%) showed deficient mismatch repair status (dMMR). Most of them were found in the M0 group $(N=4 / 82(4.9 \%))$ followed by the $\operatorname{HEP}(N=2 /$ $82(2.4 \%))$ and the PUL group $(N=1 / 82(1.2 \%))$. In the metastatic group (HEP and PUL), two out of three patients showed mutations in the $B R A F$ gene, whereas no mutations in the analyzed $R A S$ genes could be obtained. One out of two 
$B R A F$-mutated cases showed combined overexpression of CD133, the other case showed a coincidence of deregulated p53 status and deregulated CD133 status. In the M0 cohort with dMMR status, two $B R A F$-mutated cases were found, one of them with combined deregulated p53 status. One case showed exclusive $B R A F$ mutation and one case exhibited combined $\beta$-catenin and CD133 expression. One patient showed no alterations in the analyzed pathways. Taken together, no distinct pattern of altered biomarkers or biomarker combinations could be obtained and due to low patient numbers, no level of statistical significance was obtained in the global analysis $(p=0.36)$ or head-to-head comparisons (Table 3).

Multivariate analysis adjusting results for the most relevant confounders (age, lymph node status (N0 vs. N+), MAP kinase status, MMR status, p53, beta-catenin and CD133) confirmed the independent association between the investigated markers and metastatic patterns (Online Resource 5).

\section{Biomarker combinations associated with the formation of metastasis in the lungs or the liver}

When taking into account the abundance of biomarker combinations instead of single biomarkers, patients with exclusive lung metastasis (PUL group) were characterized by the presence of both a MAPK pathway mutation plus a high Wnt/ $\beta$ catenin expression $(N=23(28.0 \%))$, whereas a larger proportion of patients from the HEP and M0 group showed negativity for one or both markers (global $p=0.048$; PUL vs. HEP $p$ $=0.02$; Tables 2 and 3). Furthermore, MAPK pathway mutations in combination with high CD133 expression correlated with the development of metastasis in general compared to a non-metastatic course of the disease (M1 (PUL $N=15$ (18.3\%) and HEP $N=17(20.7 \%))$ vs. M0 $N=5$ (6.1\%); $p$ $=0.01)$ (Table 3$)$. Head-to-head comparisons of all groups according to single biomarkers and biomarker combinations as well as corresponding $p$ values are summarized in Table 3 . Frequencies and coincidence of alterations according to metastatic status are illustrated in Fig. 2a. Figure $2 \mathrm{~b}$ gives an overview of the extracted results.

\section{Survival data according to biomarker profiles}

According to biomarker profiles, survival analyses comparing overall survival (OS) and progression-free survival (PFS) of patients from the PUL versus the HEP group were performed. For OS analysis, 53 of 82 patients from the PUL group and 56 of 82 patients from the HEP group were available. For PFS, 77 of 82 patients from each the HEP and the PUL group were available.

Overall, patients with lung metastasis showed a longer OS and PFS than patients with liver metastasis (OS: 65.7 vs. 37.1 months, HR 1.41 [95\% CI: 0.96-2.06], $p=0.08$; PFS: 23.9 vs. 16.1 months, HR 1.45 [95\% CI: 1.06-2.00], $p=0.02$ ) (Online Resource 6 and Online Resource 7).

In both the PUL and the HEP group, the presence of a MAPK pathway mutation was associated with inferior OS (PUL: 51.5 vs. 83.6 months, HR 1.96 [95\% CI: 1.07-3.60], $p=0.03$; HEP: 26.4 vs. 46.3 months, HR 1.93 [95\% CI: $1.13-$ 3.29], $p=0.02$ ) and PFS (PUL: 19.0 vs. 28.8 months, HR 1.99 [95\% CI: 1.18-3.35], $p=0.01$; HEP: 10.1 vs. 19.1 months, HR 1.53 [95\% CI: 0.97-2.42], $p=0.07$ ) compared to patients without MAPK pathway mutations. This effect stayed significant when comparing patients with and without MAPK pathway mutations between groups (OS: global $p=0.004$, PFS: global $p=0.002$ ) (Online Resource 6 and Online Resource 8).

Within the PUL group, the different marker combinations consisting of MAPK pathway mutations and CD133 expression status were associated with significant differences in OS and PFS. In patients with lung metastasis and a MAPK pathway mutation plus a high CD133 expression, both OS and PFS were significantly shorter than in patients with either
Table 3 Head-to-head comparisons between groups according to single biomarkers and biomarker combinations

\begin{tabular}{|c|c|c|c|c|}
\hline & $\begin{array}{l}\text { M1 } \leftrightarrow \\
\text { M0 }\end{array}$ & $\begin{array}{l}\text { PUL } \leftrightarrow \\
\text { M0 }\end{array}$ & $\begin{array}{l}\mathrm{HEP} \leftrightarrow \\
\text { M0 }\end{array}$ & $\begin{array}{l}\text { PUL } \leftrightarrow \\
\text { HEP }\end{array}$ \\
\hline \multicolumn{5}{|l|}{ Single biomarker } \\
\hline MAP kinase mutational status & 0.15 & 0.04 & 0.64 & 0.11 \\
\hline CD133 expression & 0.03 & 0.12 & 0.02 & 0.38 \\
\hline$\beta$-catenin expression & 0.72 & 0.35 & 0.75 & 0.21 \\
\hline MMR status & 0.18 & 0.17 & 0.41 & 0.56 \\
\hline \multicolumn{5}{|l|}{ Marker combinations } \\
\hline $\begin{array}{l}\text { MAP kinase mutational status PLUS } \beta \text {-catenin } \\
\text { expression }\end{array}$ & 0.50 & 0.09 & 0.52 & 0.02 \\
\hline $\begin{array}{l}\text { MAP kinase mutational status PLUS CD133 } \\
\text { expression }\end{array}$ & 0.01 & 0.02 & 0.01 & 0.69 \\
\hline$\beta$-catenin expression PLUS CD133 expression & 0.67 & 0.80 & 0.62 & 0.81 \\
\hline
\end{tabular}

Significant $p$ values are printed in italics 
a

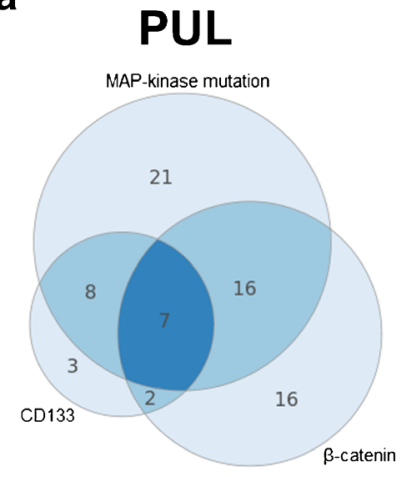

HEP

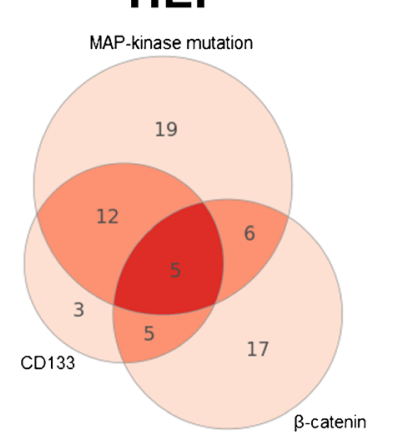

M0

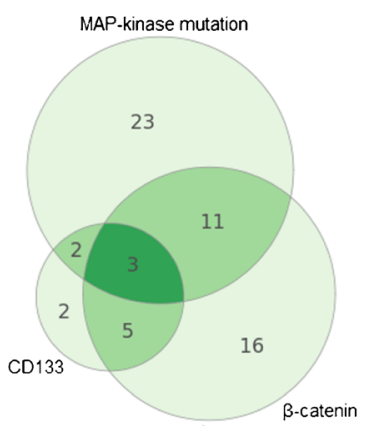

b
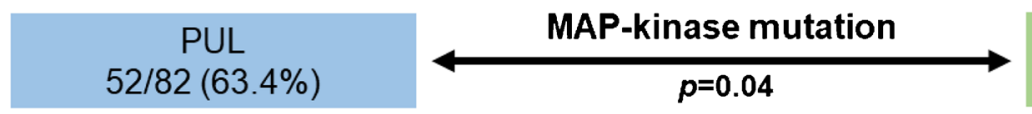

M0 $52 / 82(63.4 \%)$ $p=0.04$

$39 / 82(47.6 \%)$
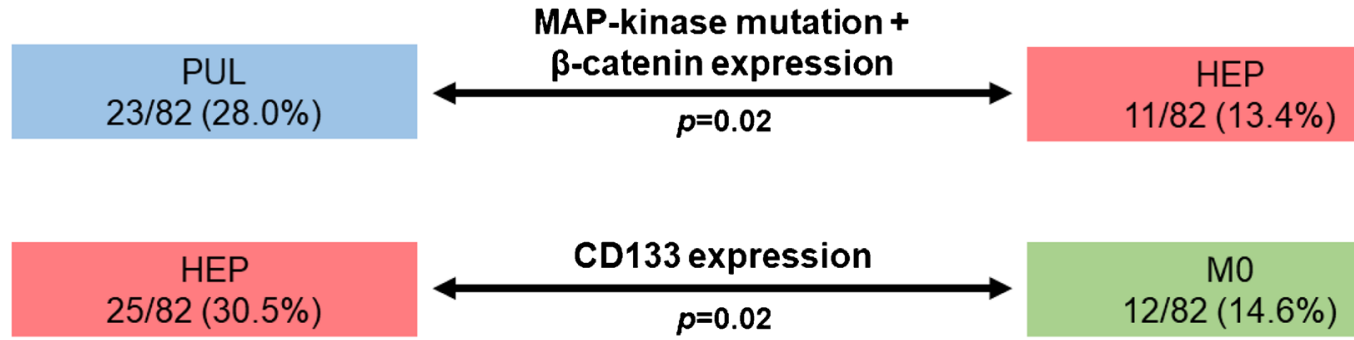

M0

$12 / 82(14.6 \%)$

Fig. 2 a Venn diagram illustrating the frequencies and coincidences of biomarker alterations according to metastatic status. Numbers indicate proven alterations which are defined as the presence of a mutation in the MAP kinase pathway and high immunohistochemical expression of

MAP kinase wild-type tumours and/or low CD133 expression (OS: 48.1 vs. 67.4 months, HR 2.37 [95\% CI: 1.20-4.67], $p=$ $0.01)$; PFS: 19.0 vs. 28.5 months, HR 1.86 [95\% CI: $1.01-$ 3.43], $p=0.04$ ) (Online Resource 6).

No survival differences were observed when focusing on CD133 and $\beta$-catenin expression as single biomarkers. Also, neither the combination of MAPK pathway mutations plus $\beta$ catenin expression status nor the combination of $\beta$-catenin plus CD133 expression status revealed any survival differences within or between groups.

\section{Discussion and conclusion}

There is consensus that colorectal cancer is a heterogeneous disease. Also, it is widely accepted that tumour biology impacts treatment efficacy and long-term outcome [24-26]. However, when it comes to different metastatic phenotypes, no reliable biomarkers predicting the formation of distant metastasis exist so far. For clinical management, knowledge about the CRC patient's individual risk to metastasize and the site of distant spread could guide aggressiveness of
Wnt/ $\beta$-catenin and CD133 (see Table 2). b Overview of the relevant biomarker alterations correlating with the formation of metastasis in the lungs and/or the liver

(local) treatment and help to stratify patients for personalized treatment approaches.

Thus, the present study aimed to investigate biomarker profiles that could predict the development of metastasis in the lungs or the liver, both pivotal organs and frequently affected by CRC metastasis. Indeed, we were able to identify biomarker profiles that are associated with different patterns of distant spread in colorectal cancer. We show that the presence of a MAPK pathway mutation plus a deregulation of the $\mathrm{Wnt} / \beta$-catenin pathway is associated with metastasis to the lungs indicating that this biomarker combination could predict a higher risk of lung metastasis. In contrast, the expression of the stem cell-associated marker CD133 correlated with the presence of liver metastasis.

The clinical interest to estimate the likelihood of lung or liver metastasis or a non-metastatic course of disease clearly lies in follow-up care after local treatment of CRC and early detection of potentially resectable or locally treatable organ metastasis. Referring to an easy-to-practice biomarker profile that predicts the development of metastasis in the liver or the lungs could enable physicians to perform diagnostic procedures according to the patient's individual risk and pave the way for early treatment of organ metastasis. With the MAPK 
pathway mutations, $\beta$-catenin as a representative from the $\mathrm{Wnt} / \beta$-catenin-pathway as well as the stem cell marker CD133, we specifically examined standard biomarkers that are well established and widely used in routine diagnostics of CRC specimens.

To accomplish the highest statistical accuracy, we chose a matched-pair analysis and therefore created a biobank containing clinical data and tumour tissue specimen from 246 CRC patients with exclusive lung metastasis (PUL group), exclusive liver metastasis (HEP group) or non-metastatic disease (M0 group). Using this case-control design, patients were matched according to relevant baseline characteristics as appropriate for a matched-pair analysis to exclude confounding factors.

With regard to the biological background, CRC develops as a result of different combinations of genetic alterations, epigenetic changes and posttranslational modifications [27]. Most CRC arises through the gradual malignant progression of a benign precursor lesion known as an adenoma. The majority of sporadic CRC arises via the adenoma-carcinoma sequence starting with mutations of the tumour-suppressor adenomatous polyposis coli (APC) with subsequent dysregulation of the $\mathrm{Wnt} / \beta$-catenin pathway [27]. Forced expression of mutant KRAS in CRC enhances nuclear $\beta$-catenin accumulation and increases the levels of Wnt target genes. This KRAS-induced augmentation of $\mathrm{Wnt} / \beta$ catenin activity results in increased proliferation and malignant progression and therefore is an important driver for the formation of metastases [28, 29].

In our study, we demonstrate that a combined expression of $\beta$-catenin together with a MAPK pathway mutation correlates with metastasis to the lungs but not to the liver. Indeed, some clinical studies described MAP kinase mutations as independent risk factors for the development of lung metastasis [14-17]. We focused on the metastatic route into the lungs and compared them against the liver and non-metastatic disease and can clearly disclose a difference in the formation of metastasis to these organs. Based on the results of Horst and coworkers, who showed that the enhancement of the Wnt/ $\beta$ catenin pathway leads to proliferation and malignant progression in vitro [29], we observe that this marker combination correlates with the formation of metastasis in the lungs but not in the liver.

Investigating the stem cell marker CD133, the subgroup of patients with liver metastasis showed the highest proportion of tumours with high CD133 expression compared to patients with lung metastasis or non-metastatic disease. In detail, $30.5 \%$ of patients with exclusive liver metastasis showed CD133 positivity and the percentage was comparable to previously reported results from Horst et al. that described $26 \%$ of unselected colorectal tumours as CD133 high and $74 \%$ as CD133 low [30].

Interestingly, the combination of a MAPK pathway mutation plus a high CD133 expression was associated with a distant spread in general (M0 vs. M1). This finding may be explained by the fact that the MAPK pathway mutation still depicts the dominant driver in formation of metastasis. Also, the combination of nuclear $\beta$-catenin expression plus CD133 expression did not reveal any significant association with metastatic patterns in the present analysis. This is not necessarily in contrast to previously published data from our group showing an association between the high expression of CD133 and/ or nuclear $\beta$-catenin and a high risk of distant metastases in right-sided colon cancers [19]. As the majority ( $89 \%$ ) of primaries were left-sided in the present analysis and due to known differences between left- and right-sided colon cancers, data is neither comparable nor transferable.

Furthermore, we present survival data from our study population according to metastatic group. It is acknowledged that patients with CRC and lung metastasis live longer than those with other metastatic manifestations [31, 32]. Accordingly, in the present study, patients with lung metastasis showed longer OS and PFS than patients with liver metastasis. Furthermore, the known prognostic value of the MAPK pathway mutational status was clearly confirmed indicating that the patient cohort analyzed was representative of large CRC populations. Interestingly, no prognostic relevance of the CD133 and $\beta$ catenin expression status was observed thus seeming only predictive for the formation of metastasis but not decisive for long-term outcome.

Since most tumours of the investigated patient cohort derived from the left-sided colon and rectum, as expected, only few patients $(2.9 \%)$ showed dMMR. Due to low numbers, statistical comparisons are not reasonable and the relevance of the MMR status stays unclear for the investigated questions of the present analysis. In the investigated study cohort, patients with primary tumours in the rectum were overrepresented in both metastatic groups (PUL and HEP) compared to the control group (M0). However, the in-depth analysis revealed no statistical difference between the PUL and the HEP group. Thus, results showing significant differences in singlebiomarker alterations as well as biomarker combinations between these groups appear not to be biased by localization of the primary tumour. Furthermore, multivariate analysis ruled out confounding factors for PUL versus HEP patients.

Of interest, previous studies show that KRAS mutations are associated with metastasis to the central nervous system (CNS) $[18,23]$. Thus, CRC patients with haematogenous spread to the lungs and to the CNS seem to have molecular features in common that possibly indicate similar carcinogenic pathways leading to metastasis formation in the lungs and/or brain. However, underlying mechanisms can certainly not be derived from the presented results.

The present analysis is certainly limited by its retrospective and exploratory design as well as data acquisition from a cancer registry. Thus, by its nature, the study cannot serve with results from functional studies or in vivo experiments but 
nevertheless provides interesting information about the presented biomarker alterations.

Taken together, our data indicate that the enhanced activity of the Wnt/ $\beta$-catenin pathway intensified by a MAPK pathway mutation may play a crucial role in the formation of lung metastasis, whereas high expression of CD133 correlates with the presence of liver metastasis. Based on these findings, we raise the hypothesis that the anatomic site of metastasis formation may depend on different patterns or varying sequences of molecular marker alterations during CRC carcinogenesis, respectively. On this basis, we would like to initiate a discussion on the necessity of a novel and clinically relevant classification of CRC. Certainly, precise mechanisms underlying the metastasis formation in different anatomic still remain unclear. However, knowledge of the anticipated site of distant metastasis would substantially impact clinical management, so an increased effort into the identification of solid biomarkers for organotropic formation of metastasis is justified.

Supplementary Information The online version contains supplementary material available at https://doi.org/10.1007/s00428-020-02983-6.

Acknowledgments This work is part of the doctoral thesis of Marianne Ehrnsperger, Thomas Schneider and Francesco Taverna. We thank all patients and their families for their contribution to our research.

Authors' contributions M.M. and J.N. performed literature research, collected data and wrote the manuscript. F.T. and J.N. performed immunohistochemical staining and analysis. F.T. and J.K. performed mutation analyses. F.T., M.M. and J.N. performed statistical analysis of data and designed tables and figures. T.S., V.H., J.E. and T.K. reviewed the manuscript critically and provided valuable expertise for medical writing.

Funding Open Access funding enabled and organized by Projekt DEAL. During the creation of the presented data, Marlies Michl was granted by the "Scholarship for excellent young scientists as part of the Bavarian Equal Opportunities Fund". The study was financed by the Association for the Promotion of Science and Research at the Medical Faculty of the Ludwig-Maximilians-University Munich.

Data Availability Data that support the findings of this study is available from the corresponding author (J.N.) upon reasonable request.

\section{Compliance with ethical standards}

Conflict of interest Marlies Michl received honoraria for talks by SIRTeX, Roche and MSD and travel expenses by SIRTeX, Amgen and Merck. Joerg Kumbrink received honoraria and reimbursement for travel and accommodation from AstraZeneca, Novartis, Quality Initiative in Pathology (QuIP) and Roche. Francesco Taverna and Jutta Engel declare no conflict of interest. Volker Heinemann received honoraria for talks, advisory boards and travel expenses by Merck, Amgen, Roche, Takeda, Servier, Pierre Fabre, Taiho, Lilly Oncology, Servier, Sanofi and Bayer Pharmaceuticals. Thomas Kirchner received honoraria for Consulting/ Advisory by Amgen, AstraZeneca, Merck KGaA, MSD, Novartis, Pfizer and Roche, for Research Funding by Merck and Roche; for talks by Merck, Astra Zeneca. Jens Neumann received honoraria for talks by Roche, Pfizer, MSD, Pierre Fabre and Falk.
Ethics approval The study was approved by the local ethical review committee (approval number 18-105) and conducted according to the Declaration of Helsinki.

Open Access This article is licensed under a Creative Commons Attribution 4.0 International License, which permits use, sharing, adaptation, distribution and reproduction in any medium or format, as long as you give appropriate credit to the original author(s) and the source, provide a link to the Creative Commons licence, and indicate if changes were made. The images or other third party material in this article are included in the article's Creative Commons licence, unless indicated otherwise in a credit line to the material. If material is not included in the article's Creative Commons licence and your intended use is not permitted by statutory regulation or exceeds the permitted use, you will need to obtain permission directly from the copyright holder. To view a copy of this licence, visit http://creativecommons.org/licenses/by/4.0/.

\section{References}

1. Qiu M, Hu J, Yang D, Cosgrove DP, Xu R (2015) Pattern of distant metastases in colorectal cancer: a SEER based study. Oncotarget 6: 38658-38666. https://doi.org/10.18632/oncotarget.6130

2. Mitry E, Guiu B, Cosconea S, Jooste V, Faivre J, Bouvier AM (2010) Epidemiology, management and prognosis of colorectal cancer with lung metastases: a 30-year population-based study. Gut 59:1383-1388. https://doi.org/10.1136/gut.2010.211557

3. Haussmann J, Matuschek C, Bölke E, Orth K, Ghadjar P, Budach W (2019) The role of local treatment in oligometastatic and oligoprogressive cancer. Dtsch Arztebl Int 116(50):849-856. https://doi.org/10.3238/arztebl.2019.0849

4. Van Cutsem E, Cervantes A, Adam R, Sobrero A, van Krieken JH, Aderka D, Aranda Aguilar E, Bardelli A, Benson A et al (2016) ESMO consensus guidelines for the management of patients with metastatic colorectal cancer. Ann Oncol 27:1386-1422. https://doi. org/10.1093/annonc/mdw235

5. Tomlinson JS, Jarnagin WR, DeMatteo RP, Fong Y, Kornprat P, Gonen M, Kemeny N, Brennan MF, Blumgart LH, D'Angelica M (2007) Actual 10-year survival after resection of colorectal liver metastases defines cure. J Clin Oncol 25:4575-4580. https://doi. org/10.1200/JCO.2007.11.0833

6. Pfannschmidt J, Dienemann H, Hoffmann H (2007) Surgical resection of pulmonary metastases from colorectal cancer: a systematic review of published series. Ann Thorac Surg 84:324-338

7. Fong Y, Fortner J, Sun RL, Brennan MF, Blumgart LH (1999) Clinical score for predicting recurrence after hepatic resection for metastatic colorectal cancer: analysis of 1001 consecutive cases. Ann Surg 230:309-318. https://doi.org/10.1097/00000658199909000-00004

8. Meimarakis G, Spelsberg F, Angele M, Preissler G, Fertmann J, Crispin A, Reu S, Kalaitzis N, Stemmler M, Giessen C, Heinemann V, Stintzing S, Hatz R, Winter H (2014) Resection of pulmonary metastases from colon and rectal cancer: factors to predict survival differ regarding to the origin of the primary tumor. Ann Surg Oncol 21:2563-2572. https://doi.org/10.1245/s10434-014-3646-1

9. Stintzing S, Miller-Phillips L, Modest DP, Fischer von Weikersthal L, Decker T, Kiani A, Vehling-Kaiser V, Al-Batran S-E, Heintges $\mathrm{T}$, Kahl C et al (2017) Impact of BRAF and RAS mutations on firstline efficacy of FOLFIRI plus cetuximab versus FOLFIRI plus bevacizumab: analysis of the FIRE-3 (AIO KRK-0306) study. Eur J Cancer 79:50-60. https://doi.org/10.1016/j.ejca.2017.03.023

10. Sorich MJ, Wiese MD, Rowland A, Kichenadasse G, McKinnon RA, Karapetis CS (2015) Extended RAS mutations and anti-EGFR monoclonal antibody survival benefit in metastatic colorectal 
cancer: a metaanalysis of randomized, controlled trials. Ann Oncol 26:13-21. https://doi.org/10.1093/annonc/mdu378

11. Modest DP, Ricard I, Heinemann V, Hegewisch-Becker S, Schmiegel W, Porschen R, Stintzing S, Graeven U, Arnold D, von Weikersthal LF, Giessen-Jung C, Stahler A, Schmoll HJ, Jung A, Kirchner T, Tannapfel A, Reinacher-Schick A (2016) Outcome according to KRAS-, NRAS- and BRAF-mutation as well as KRAS mutation variants: pooled analysis of five randomized trials in metastatic colorectal cancer by the AIO colorectal cancer study group. Ann Oncol 27:1746-1753. https://doi.org/10. 1093/annonc/mdw261

12. Andre T, Shiu K-K, Kim TW et al Pembrolizumab vs chemotherapy for microsatellite instability-high/mismatch repair deficient metastatic colorectal cancer: The phase 3 KEYNOTE-177 study. J Clin Oncol 38: 2020 (suppl; abstr LBA4). https://doi.org/10.1200/ JCO.2020.38.18_suppl.LBA4

13. Lipsyc MD, Yaeger R (2015) Impact of somatic mutations on patterns of metastasis in colorectal cancer. J Gastrointest Oncol 6:645649. https://doi.org/10.3978/j.issn.2078-6891.2015.045

14. Pereira AA, Rego JF, Morris V, Overman MJ, Eng C, Garrett CR, Boutin AT, Ferrarotto R, Lee M, Jiang Z-Q, Hoff PM, Vauthey J-N, Vilar E, Maru D, Kopetz S (2015) Association between KRAS mutation and lung metastasis in advanced colorectal cancer. Br J Cancer 112:424-428. https://doi.org/10.1038/bjc.2014.619

15. Tie J, Lipton L, Desai J, Gibbs P, Jorissen RN, Christie M, Drummond KJ, Thomson BNJ, Usatoff V, Evans PM, Pick AW, Knight S, Carne PWG, Berry R, Polglase A, McMurrick P, Zhao Q, Busam D, Strausberg RL, Domingo E, Tomlinson IPM, Midgley R, Kerr D, Sieber OM (2011) KRAS mutation is associated with lung metastasis in patients with curatively resected colorectal cancer. Clin Cancer Res 17:1122-1130. https://doi.org/10.1158/10780432.CCR-10-1720

16. Yaeger R, Cowell E, Chou JF, Gewirtz AN, Borsu L, Vakiani E, Solit DB, Rosen N, Capanu M, Ladanyi M, Kemeny N (2015) RAS mutations affect pattern of metastatic spread and increase propensity for brain metastasis in colorectal cancer. Cancer 121:11951203. https://doi.org/10.1002/cncr.29196

17. Cejas P, Lopez-Gomez M, Aguayo C, Madero R, de Castro Carpeño J, Belda-Iniesta C, Barriuso J, Moreno García V, Larrauri J, López R, Casado E, Gonzalez-Barón M, Feliuet J (2009) KRAS mutations in primary colorectal cancer tumors and related metastases: a potential role in prediction of lung metastasis. PLoS One 4:e8199. https://doi.org/10.1371/journal.pone.0008199

18. El-Deiry WS, Vijayvergia N, Xiu J, Scicchitano A, Lim B, Yee NS, Harvey HA, Gatalica Z, Reddy S (2015) Molecular profiling of 6, 892 colorectal cancer samples suggests different possible treatment options specific to metastatic sites. Cancer Biol Ther 16(12):17261737. https://doi.org/10.1080/15384047.2015.1113356

19. Neumann J, Horst D, Kriegl L, Maatz S, Engel J, Jung A, Kirchner $\mathrm{T}$ (2012) A simple immunohistochemical algorithm predicts the risk of distant metastases in right-sided colon cancer. Histopathology 60:416-426. https://doi.org/10.1111/j.1365-2559. 2011.04126.x

20. Horst D, Reu S, Kriegl L, Engel J, Kirchner T, Jung A (2009) The intratumoral distribution of nuclear beta-catenin is a prognostic marker in colon cancer. Cancer 115:2063-2070. https://doi.org/ 10.1002/cncr.24254

21. Horst D, Scheel SK, Liebmann S, Neumann J, Maatz S, Kirchner T, Jung A (2009) The cancer stem cell marker CD133 has high prognostic impact but unknown functional relevance for the metastasis of human colon cancer. J Pathol 219:427-434. https://doi.org/ 10.1002/path.2597

22. Neumann J, Löhrs L, Albertsmeier M, Reu S, Guba M, Kirchner T, Angele M (2015) The stem cell associated biomarker CD133 is associated with distant hematogeneous liver metastases but not with peritoneal carcinomatosis in colorectal cancer. Cancer Investig 33(8):354-360. https://doi.org/10.3109/07357907.2015.1047507

23. Michl M, Heinemann V, Jung A, Kirchner T, Neumann J (2015) Expression of stemness associated markers correlates with distant spread to the liver but not to with brain metastases in colorectal cancer. Pathol Res Pract 211(8):601-609. https://doi.org/10.1016/ j.prp.2015.05.006

24. Yamashita S, Chun YS, Kopetz SE, Vauthey JN (2018) Biomarkers in colorectal liver metastases. Br J Surg 105:618-627. https://doi. org/10.1002/bjs. 10834

25. Patel JN, Fong MK, Jagosky M (2019) Colorectal cancer biomarkers in the era of personalized medicine. J Pers Med 9:3. https://doi.org/10.3390/jpm9010003

26. Taieb J, Jung A, Sartore-Bianchi A, Peeters M, Seligmann J, Zaanan A, Burdon P, Montagut C, Laurent-Puig P (2019) The evolving biomarker landscape for treatment selection in metastatic colorectal cancer. Drugs 79:1375-1394. https://doi.org/10.1007/ s40265-019-01165-2

27. Fearon ER (2011) Molecular genetics of colorectal cancer. Annu Rev Pathol 6:479-507. https://doi.org/10.1146/annurev-pathol011110-130235

28. Janssen KP, Alberici P, Fsihi H, Gaspar C, Breukel C, Franken P, Rosty C, Abal M, El Marjou F, Smits R, Louvard D, Fodde R, Robine S (2006) APC and oncogenic KRAS are synergistic in enhancing Wnt signaling in intestinal tumor formation and progression. Gastroenterology 131:1096-1109. https://doi.org/10.1053/j. gastro.2006.08.011

29. Horst D, Chen J, Morikawa T, Ogino S, Kirchner T, Shivdasani RA (2012) Differential WNT activity in colorectal cancer confers limited tumorigenic potential and is regulated by MAPK signaling. Cancer Res 72:1547-1556. https://doi.org/10.1158/0008-5472. CAN-11-3222

30. Horst D, Kriegl L, Engel J, Kirchner T, Jung A (2008) CD133 expression is an independent prognostic marker for low survival in colorectal cancer. Br J Cancer 99:1285-1289. https://doi.org/ 10.1038/sj.bjc. 6604664

31. Koehne CH, Cunningham D, Di Costanzo F, Glimelius B, Blijham G, Aranda E, Scheithauer W, Rougier P, Palmer M, Wils J, Baron B, Pignatti F, Schöffski P, Micheel S, Hecker H (2002) Clinical determinants of survival in patients with 5-fluorouracil-based treatment for metastatic colorectal cancer: results of a multivariate analysis of 3825 patients. Ann Oncol 13:308-317. https://doi.org/10. 1093/annonc/mdf034

32. Khattak MA, Martin HL, Beeke C, Price T, Carruthers S, Kim S, Padbury R, Karapetis CS (2012) Survival differences in patients with metastatic colorectal cancer and with single site metastatic disease at initial presentation: results from South Australian clinical registry for advanced colorectal cancer. Clin Colorectal Cancer 11: 247-254. https://doi.org/10.1016/j.clcc.2012.06.004

Publisher's note Springer Nature remains neutral with regard to jurisdictional claims in published maps and institutional affiliations. 